\title{
Conditions-cadres générales et explications relatives au contrat de travail
}

Etant donné que, depuis 1991, les sociétés cantonales de médecine élaborent leurs propres recommandations de salaires pour les assistantes médicales, nous renonçons désormais à publier des recommandations salariales pour l'ensemble de la Suisse.

Les conditions-cadres ci-après conservent toutefois leur valeur pour fixer individuellement le salaire d'une assistante médicale.

\section{Formation}

- Diplôme de la Fédération des médecins suisses (estampille) resp. certificat de capacité de la FMH avec certificat de radioprotection et autorisation de faire des radiographies

- Certificat fédéral de capacité d'assistante médicale

2. Conditions de travail: bases de calcul

- Semaine de 42 heures en moyenne annuelle

- 4 semaines de vacances (assistantes médicales de moins de 20 ans et de plus de 50 ans: 5 semaines).

Toute dérogation importante à ces conditions sera répercutée sur le salaire.

\section{Normes régionales}

Les salaires doivent être adaptés aux conditions régionales.

\section{4. $13^{\mathrm{e}}$ salaire}

Un $13^{\mathrm{e}}$ salaire doit être versé à l'assistante médicale à la fin de l'année. Si les rapports de service n'ont pas duré pendant toute l'année civile, le $13^{\mathrm{e}}$ salaire sera versé au prorata des mois d'engagement.

\section{Primes d'ancienneté et augmentations}

\section{du salaire réel}

Le montant d'une prime d'ancienneté devrait figurer, également en 2013, parmi les points discutés au cours de l'entretien annuel sur les qualifications de l'employée. Au moment de définir le salaire, il convient notamment de prendre aussi en considération toute éventuelle fonction supplémentaire (par ex. formation des personnes en apprentissage).

\section{Compensation du renchérissement} (www.bfs.admin.ch)

Le renchérissement devrait être compensé. Lorsque la compensation du renchérissement est convenue par contrat, elle doit être accordée en tous les cas. Si le renchérissement est négatif, ce dernier ne doit pas être compensé (ISPC septembre 2013: $-0,1 \%$; octobre 2013: $-0,3 \%$, indice de base: décembre 2010).

\section{Travail à temps partiel rétribué au mois}

Pour le travail à temps partiel, le salaire brut recommandé s'élève au 1/42 du salaire brut entier multiplié par le nombre des heures de travail hebdomadaire convenu (base de calcul: 42 heures hebdomadaires).

\section{Salaire horaire}

Un salaire horaire est recommandé en cas d'horaire de travail très réduit et en même temps irrégulier. Le taux horaire conseillé est de $6 \%$ du salaire mensuel pour une activité à temps complet (le $13^{\text {e }}$ salaire y étant inclus au prorata). Il sera complété par une contribution aux vacances de $8,33 \%$ correspondant aux quatre semaines de vacances habituelles (pour 5 semaines le taux sera de 10,64\% et pour 6 semaines 13,04\% etc.). Ce mode de calcul est également valable pour le paiement des heures supplémentaires.

\section{Heures supplémentaires}

\section{(chiffre 2 du contrat type)}

Dans la mesure du possible, les heures supplémentaires doivent être compensées par des vacances ou du temps libre d'une même durée. Si ce n'est pas possible, on peut désormais choisir parmi les variantes suivantes:

- L'indemnisation des heures supplémentaires par une majoration de salaire de $25 \%$ pour une activité à temps complet ou à temps partiel (préconisée par la FMH et la FSAAM).

- Dans le cas d'une activité à temps partiel, les heures supplémentaires sont indemnisées selon le taux horaire normal dans la mesure où elles ne dépassent pas la durée du temps de travail réglementaire d'une assistante à temps plein au cabinet médical (préconisée par la SVA). 


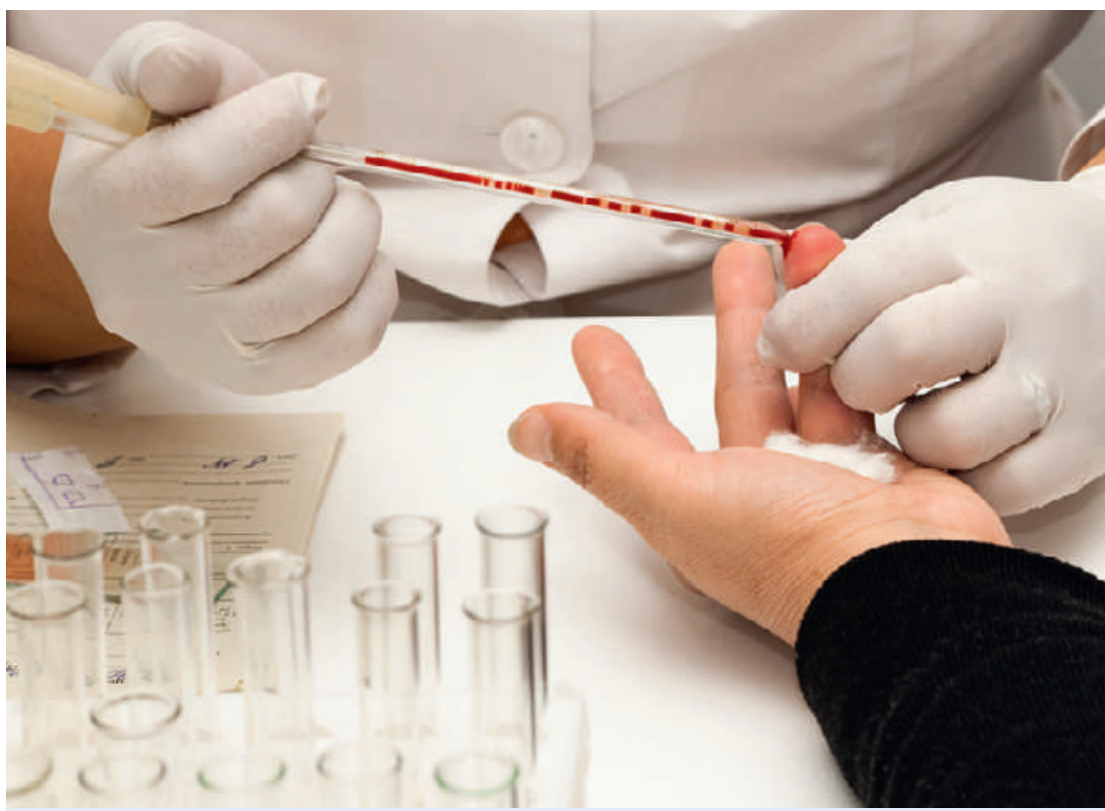

Les salaires des assistantes médicales doivent être adaptés aux conditions régionales.
- Fédération des médecins suisses (FMH), Elfenstrasse 18, case postale 300, 3000 Berne 15, tél. 03135911 11, www.fmh.ch, courriel: mpa[at] fmh.ch

- Association romande des assistantes médicales (ARAM), 1003 Lausanne, tél. 0793801244 / 077 43679 27, www.aram-vd.ch, courriel: info[at] aram-vd.ch

- Association suisse des assistantes médicales (SVA), case postale 6432, 3001 Berne, tél. 031/ 3805454 www.sva.ch, courriel: sekretariat[at] sva.ch

- Berufsverband Medizinischer Praxisassistentinnen (BMPA), Obergrundstrasse 65, $6003 \mathrm{Lu}$ cerne, tél. 04131011 21, www.bmpa.ch, courriel: sekretariat[at]bmpa.ch

- Fédération suisse des associations d'assistantes médicales (FSAAM), case postale, 3051 Bienne, tél. 07967454 71, www.fsaam.ch, courriel: sekreta riat[at]bsmpa-fsaam.ch

- Association genevoise des assistantes médicales (AGAM), 1200 Genève, www.agam-ge.ch, courriel: presidente[at]agam-ge.ch

- D'un commun accord écrit, l'employeur et l'assistante médicale peuvent choisir une autre réglementation.

Le taux horaire est de $6 \%$ d'un plein salaire mensuel pour une activité à temps complet, auquel s'ajoute la contribution aux vacances (cf. chiffre 8 , «salaire horaire»).

\section{Les dispositions et recommandations suivantes s'appliquent à tous les salaires:}

1. Déductions sur le salaire brut

- AVS, AI, APG, ACI: 6,25\% (AVS, AI, APG = 5,15\%, $\mathrm{ACI}=1,1 \%)$

- Assurance-accidents non professionnels (pour un engagement de plus de 8 heures par semaine): déduction selon le contrat d'assurance conclu

- Prévoyance professionnelle (2e pilier LPP): part de l'employée à la contribution (habituellement $50 \%$ ) calculée en fonction de l'âge, selon le certificat d'assurance.

2. Contrats de travail et autres conditions d'engagement

Nous préconisons d'établir le contrat par écrit, sur la formule de la FMH créée conjointement avec les associations des assistantes médicales, accompagnée des recommandations cantonales. Ces documents peuvent être obtenus aux adresses suivantes:
L'Association des assistantes médicales du Tessin a sa propre convention de travail ou contrat de travail; son adresse est la suivante:

- Associazione Ticinese Assistenti di studio Medico (ATAM), c/o M. Carrera, Via dei Faggi 2 A, 6912 Lugano-Pazzallo, www.atam.ch, courriel: atam[at]bluewin.ch

Les salaires des personnes en formation se basent sur les recommandations cantonales.

Délégués aux questions concernant les assistantes médicales:

Dr Adrian Sury, président

Dr Renato Tognino, vice-président

Pour la Fédération suisse des associations des assistantes médicales FSAAM (AGAM, ATAM, BMPA): Mme Elwina Kaufmann, présidente

Pour l'Association Romande des Assistantes Médicales ARAM:

Anne-Claude Perrette et Marie-Paule Fauchère, présidentes

Pour l'Association suisse des assistantes médicales SVA: Me Bruno Gutknecht, secrétaire central 